Surgery for

Congenital

Heart Disease

\title{
Conventional and sutureless techniques for management of the pulmonary veins: Evolution of indications from postrepair pulmonary vein stenosis to primary pulmonary vein anomalies
}

Tae-Jin Yun, MD

John G. Coles, MD

Igor E. Konstantinov, MD, PhD

Osman O. Al-Radi, MD

Rachel M. Wald, MD

Vitor Guerra, MD

Nilto C. de Oliveira, MD

Glen S. Van Arsdell, MD

William G. Williams, MD

Jeffrey Smallhorn, MD

Christopher A. Caldarone, MD

From the Division of Cardiovascular Surgery and Cardiology at The Hospital for Sick Children and University of Toronto, Toronto, Ontario, Canada.

Read at the Eighty-fourth Annual Meeting of The American Association for Thoracic Surgery, Toronto, Ontario, Canada, April 25-28, 2004.

Received for publication April 25, 2004; revisions received Aug 20, 2004; accepted for publication Aug 25, 2004.

Address for reprints: Christopher Caldarone, MD, Division of Cardiovascular Surgery, The Hospital for Sick Children, 555 University Ave, Toronto, Ontario, Canada, M5G 1X8 (E-mail: christopher.caldarone@ sickkids. ca).

J Thorac Cardiovasc Surg 2005;129:167-74 $0022-5223 / \$ 30.00$

Copyright (c) 2005 by The American Association for Thoracic Surgery

doi:10.1016/j.jtcvs.2004.08.043

Objective: We have previously reported a limited but favorable experience with a novel sutureless technique for surgical management of postoperative pulmonary vein stenosis occurring after repair of total anomalous pulmonary venous drainage. Because this technique requires integrity of the retrocardiac space for hemostasis, extension of the technique to the primary repair of pulmonary vein anomalies requires evaluation. This analysis reviews our experience with the sutureless technique in patients with postrepair pulmonary vein stenosis, as well as our extension of the technique into primary repair of pulmonary vein anomalies.

Methods: Retrospective univariable-multivariable analysis of all pulmonary vein stenosis procedures and sutureless pulmonary vein procedures over a 20 -year period was performed. Cox proportional hazards modeling was used to identify variables associated with freedom from reoperation or death.

Results: Sixty patients underwent 73 procedures, with pulmonary vein stenosis present in 65 procedures. The sutureless technique was used in 40 procedures. Freedom from reoperation or death at 5 years after the initial procedure was $49 \%$. Unadjusted freedom from reoperation or death was greater with the sutureless technique for patients with postrepair pulmonary vein stenosis $(P=.04)$. By using multivariable analysis, a higher pulmonary vein stenosis score was associated with greater risk of reoperation or death. After adjustment, the sutureless repair was associated with a nonsignificant trend toward greater freedom from reoperation or death $(P=.12)$. Despite the absence of retrocardiac adhesions, operative mortality was not increased with the sutureless technique $(P=$ .64). Techniques to control bleeding (intrapleural hilar reapproximation) and improve exposure (inferior vena cava division) were identified.

Conclusion: The sutureless technique for postrepair pulmonary vein stenosis is associated with encouraging midterm results. Extension of the indications for the technique to primary repair appears safe with the development of simple intraoperative maneuvers. 


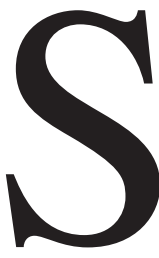

urgical relief of pulmonary vein stenosis (PVS) is a difficult clinical problem associated with significant risk of recurrent obstruction or death, requiring reoperation for stenosis and, in some reports, lung transplantation. ${ }^{1-5} \mathrm{We}$ and others have previously reported a management strategy for PVS that is based on the use of a sutureless technique of pulmonary vein anastomosis. ${ }^{2,4,6-8}$ Conceptually, the technique is based on the premise that anastomosis of the left atrium to the pericardium surrounding the divided edges of the pulmonary veins (rather than the edges of the pulmonary veins) will prevent geometric distortion of the suture line, reduce local turbulence, and prevent a subsequent spiral of intimal hypertrophy and progressive local distortion. ${ }^{9,10}$

The efficacy of the sutureless technique in prevention of recurrent PVS is not well established. Furthermore, because the technique relies on the integrity of the retrocardiac pericardium for hemostasis, the applicability of this technique to patients without previous cardiac surgery (ie, absence of pericardial thickening and adhesions) is also not well established. In the present report our experience with PVS is reviewed, with the objectives of identifying risk factors predictive of reoperation or death with various management strategies and evaluating technical problems associated with the sutureless technique.

\section{Methods}

At the Hospital for Sick Children in Toronto, 60 patients underwent operative intervention for PVS between February 1983 and October 2003. Institutional review board approval was obtained, and demographic, operative, and postoperative records and echocardiographic data were analyzed. Follow-up was $88 \%$ complete (patient status current to October 2003).

\section{PVS Score}

Operative reports and echocardiographic data were reviewed to grade the severity of PVS in an individual patient, and the reported degree of stenosis in each pulmonary vein was graded from 0 to 3 ( 0 , no stenosis; 1 , mild stenosis; 2 , severe stenosis; 3 , occluded). The sum of these scores was defined as the PVS score (possible range, 0-12).

\section{Statistical Analysis}

Data are presented as frequencies, medians with ranges, or means with SDs. Survival data are represented by using the Kaplan-Meier method. Stratified survival curves were plotted to explore unadjusted group differences for variables of interest. A Cox proportional hazards model was fitted with time to death or reoperation as the dependent variable and stratified by indication categories (described below) to allow different baseline hazards for each category. Variable selection was backward elimination with bootstrapping. The assumption of proportional hazards was tested by using an interaction term between time and each variable in the final model. The final model validation (to assess for overfitting) and calibration was done by means of bootstrapping. Analyses were conducted by using S-plus 6.1 (Insightful Corp) and Statistica analysis software version 5.5 (StatSoft, Inc).

\section{Results \\ Patients}

The breakdown of the patient population is shown in Figure 1. The cohort could be categorized into 4 groups (indication categories) on the basis of the status of the pulmonary veins (Table 1). Seventeen patients had PVS that developed after repair of total anomalous pulmonary venous drainage (postrepair PVS). Thirty-six patients underwent repair of PVS in the setting of naive pulmonary veins, which was defined as the absence of any previous operative procedure specifically dedicated to the pulmonary veins. Within this group, 10 patients had a variety of (nonpulmonary vein) prior cardiac surgical procedures (postoperative PVS), and 26 patients had PVS in the absence of any prior cardiac surgery (unoperated PVS). Seven patients were believed to be at risk for PVS because of small pulmonary veins and underwent a pulmonary vein enlarging procedure in association with another operative procedure (small PV). The size of the individual pulmonary veins adjusted to body surface area for the small pulmonary vein group was 30 to $46 \mathrm{~mm} / \mathrm{m}^{2}$, which is within a range previously reported to be at high risk for PVS, death, or both. ${ }^{11}$

Among the 18 patients who died after various operations on the pulmonary veins, $16(89 \%)$ showed signs of pulmonary venous obstruction before they died.

\section{Procedures}

Seventy-three procedures for PVS are shown in Figure 2. The sutureless repair technique was used in 35 patients as an initial procedure and in 5 patients as a second procedure. Catheterization laboratory and operative stenting procedures were often accompanied by multiple balloon dilations within the pulmonary venous system. Atrial patch procedures (atrial pulmonary venoplasty) were modifications of the procedure described by Pacifico and colleagues. ${ }^{12}$

\section{Atretic Pulmonary Veins}

The sutureless technique was used in 5 patients with atretic veins. On immediate postoperative follow-up, all but one patient had pulmonary vein patency. Three $(60 \%)$ of the 5 patients, however, died 2 to 4 months after the operation, and in the remaining 2 patients, only one had patency of the atretic pulmonary vein.

\section{Incision Versus Excision of the Pulmonary Veins}

The decision to excise or incise the stenotic portion of the pulmonary vein was made on the basis of surgeon discretion at the time of the operation rather than an institutional strategy. We tried to excise as much fibrotic tissue as possible when it subjectively appeared to have proliferative potential. In the absence of stenosis in the pulmonary veins themselves (eg, patients with postrepair PVS in the anasto- 


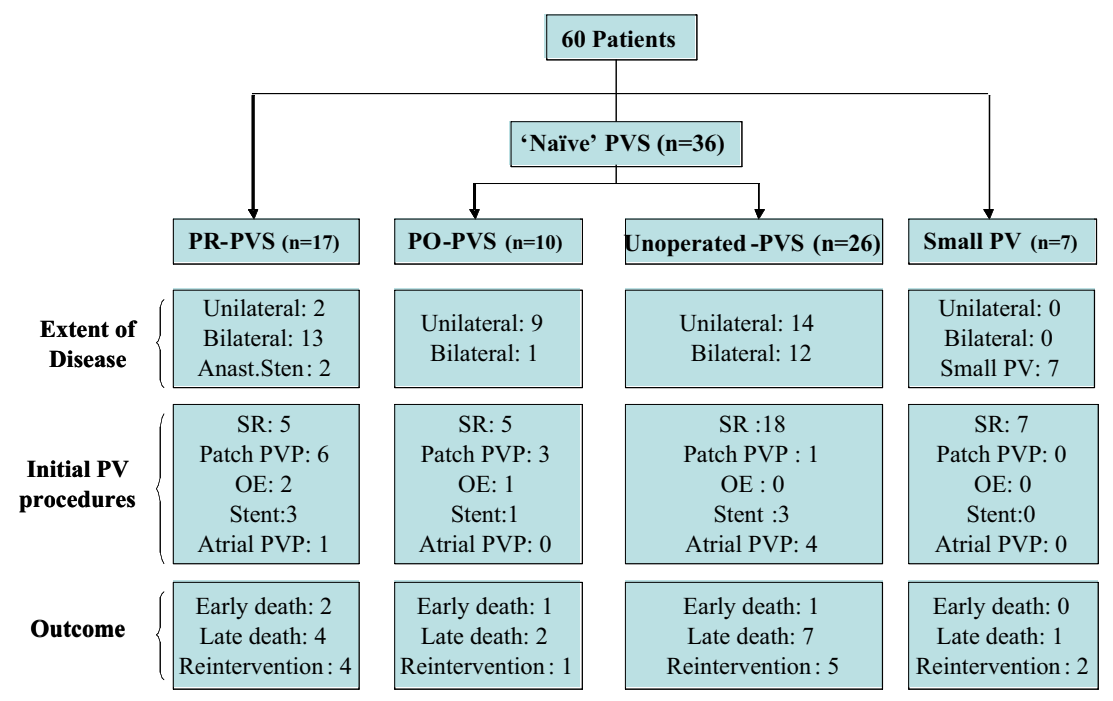

Figure 1. Breakdown of patient cohort by indication category. PR-PVS, Postrepair pulmonary vein stenosis; PO-PVS, postoperative pulmonary vein stenosis (previous nonpulmonary vein cardiac surgery); Unoperated-PVS, no previous cardiac surgery; Small PV, small pulmonary veins considered at risk for future pulmonary vein stenosis; Anast. Sten., anastomotic stenosis; $S R$, sutureless repair; PVP, Pulmonary venoplasty; $O E$, ostial endovenectomy.

motic site with normal-looking individual veins), we incise the veins rather than excise them.

\section{Postrepair PVS}

Seventeen patients with postrepair PVS underwent 24 procedures ( 7 sutureless repairs and 17 cases of conventional management). When used as the initial procedure for postrepair PVS, the sutureless technique was associated with unadjusted decreased risk of reoperation or death (univariable $P=.04$, Figure 3 ), with no reoperations or death in the sutureless group (mean follow-up, 3.1 years).

\section{Sutureless Repair as a Primary Procedure}

The sutureless technique was used as a primary procedure (eg, no previous cardiac operations) in 25 patients with PVS $(n=18)$ or at increased risk of PVS $(n=7)$. Despite the absence of retrocardiac adhesions, operative mortality was not increased compared with that of the patients who had a sutureless repair as a reoperation $(P=.64)$.

\section{Technical Problems and Maneuvers}

Intrapleural hilar reapproximation. When the sutureless technique was used in patients without previous cardiac operations, longitudinal division of the pulmonary veins into the pulmonary hilum was associated with violation of the thin pleura at the junction between the parietal and visceral pleura overlying the pulmonary hilum and left atrial bleeding into the pleural space. This was managed by means of division of the pericardium and pleura at the level of the diaphragm from anterior to posterior to the level of the phrenic nerve. The heart and divided pleura were retracted firmly toward the midline, exposing the anterior aspect of the pulmonary hilum. The parietal and visceral pleura can then be approximated with a fine running suture to achieve hemostasis. This maneuver was used successfully in 4 patients in whom such bleeding occurred.

Division of the inferior vena cava. Another useful technique to improve exposure included division of the inferior vena cava in 12 patients with bilateral PVS. In combination with division of the left atrium circumferentially around the pulmonary vein origins, the heart is tethered only by the superior vena cava, and the great vessel attachments exposure is markedly enhanced.

\section{Statistical Analysis}

By using the entire cohort, 1- and 5-year survival after the initial PVS procedure was $68.1 \%$ and $62.3 \%$, respectively, and freedom from reoperation or death at 1 and 5 years after the initial procedure was $58 \%$ and $49 \%$, respectively.

By using reoperation or death as an end point, there were 60 patients who had 23 events in the final survival model. No significant violations of the proportional hazards assumptions were identified. Variables included in the model included right atrial isomerism, the extent of PVS (PVS score), age, indication category (as a stratification variable), and procedure type. The PVS score was the only independent predictor of reoperation or death, with a hazard ratio of 12.9 for a 5-point change from a score of 2 to a score of 7 (range, 0-10; median, 4; $P<.0001$; Figure 4). After adjusting for PVS score and stratifying by indication category, there was a nonsignificant trend toward greater freedom 
TABLE 1. Profiles of patient groups

\begin{tabular}{|c|c|c|c|}
\hline Group & & & \\
\hline \multirow[t]{10}{*}{ PR-PVS ( $n=17)$} & Median age at TAPVD repair: & & $3 d$ \\
\hline & Median age at PR-PVS repair: & & $5.3 \mathrm{mo}$ \\
\hline & $\operatorname{Sex}(M / F)$ : & & $7 / 10$ \\
\hline & Types of TAPVD: & Supracardiac & 2 \\
\hline & & Cardiac & 5 \\
\hline & & Infracardiac & 8 \\
\hline & & Mixed & 2 \\
\hline & Functional single ventricle: & & $2 / 17$ \\
\hline & Associated anomalies: & RAI & 2 \\
\hline & & Others & 4 \\
\hline \multirow[t]{12}{*}{ PO-PVS (n = 10) } & Median age at initial cardiac operation: & & $1.7 \mathrm{mo}$ \\
\hline & Median age at PO-PVS repair: & & $6.2 \mathrm{mo}$ \\
\hline & Median interval: & & $7 \mathrm{mo}$ \\
\hline & $\operatorname{Sex}(M / F):$ & & $5 / 5$ \\
\hline & Original diagnosis: & HLHS & 4 \\
\hline & & D-TGA & 2 \\
\hline & & Others & 4 \\
\hline & Initial operation: & Atrial septectomy & 2 \\
\hline & & Heart transplant & 2 \\
\hline & & Senning & 1 \\
\hline & & Norwood & 1 \\
\hline & & Others & 4 \\
\hline \multirow[t]{9}{*}{ Unoperated PVS ( $\mathrm{n}=26$ ) } & Median age at PVS repair: & & $5.1 \mathrm{mo}$ \\
\hline & $\operatorname{Sex}(M / F)$ : & & $14 / 12$ \\
\hline & Associated anomalies: & ASD & 7 \\
\hline & & VSD & 6 \\
\hline & & AVSD & 6 \\
\hline & & PDA & 2 \\
\hline & & Scimitar syndrome & 2 \\
\hline & & TAPVD & 2 \\
\hline & & Others & 4 \\
\hline \multirow[t]{8}{*}{ Small PV $(n=7)$} & Diagnosis: & Age at repair & Procedure \\
\hline & Truncus arteriosus, TAPVD & $2 d$ & SR, truncus repair \\
\hline & CoA, ASD, TAPVD & $12 d$ & SR, CoA repair \\
\hline & HLHS & $4 \mathrm{mo}$ & SR, heart transplant \\
\hline & RAI, AVSD, PS, TAPVD & $3 \mathrm{mo}$ & SR, LMBT \\
\hline & RAI, AVSD, PS, TAPVD & $2.3 \mathrm{mo}$ & SR, BCPS \\
\hline & RAI, AVSD, PS, TAPVD & 3 mo & SR, BCPS \\
\hline & RAI, AVSD, PS, TAPVD & $8 \mathrm{mo}$ & SR \\
\hline
\end{tabular}

TAPVD, Total anomalous pulmonary venous drainage; $P R-P V S$, postrepair pulmonary vein stenosis; $R A I$, right atrial isomerism; $P O-P V S$, postoperative pulmonary vein stenosis; $H L H S$, hypoplastic left heart syndrome; $D-T G A$, dextro-transposition of the great arteries; $A S D$, atrial septal defect; $V S D$, ventricular septal defect; $A V S D$, atrioventricular septal defect; $P D A$, patent ductus arteriosus; $P V$, pulmonary veins; $S R$, sutureless repair; CoA, coarctation; $P S$, pulmonary stenosis; $L M B T$, left modified Blalock-Taussig shunt; $B C P S$, bidirectional cavopulmonary shunt.

from death or reoperation with the sutureless technique (hazard ratio, $0.47 ; P=.12$ ). When controlling for PVS score, bilateral stenosis was not a significant risk factor for reoperation or death.

Because of heterogeneity and the small number of patients, we failed to demonstrate robust model validity and calibration by bootstrapping. Therefore the results of this model might experience overfitting and should not be inter- preted in the context of hypothesis testing but rather hypothesis generation.

\section{Discussion}

PVS is a challenging problem because of the recurrent and often inexorable progression of the disease. The sutureless technique has been used in many centers for postrepair PVS, with encouraging but inconclusive results. ${ }^{4-6}$ Since our pre- 


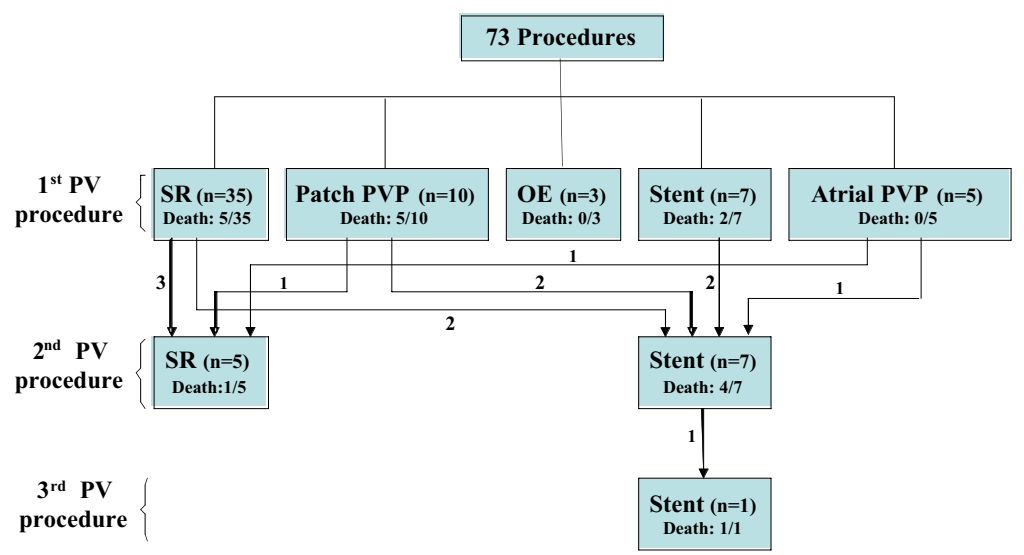

Figure 2. Breakdown of procedures performed. Abbreviations are as in the legend for Figure 1.

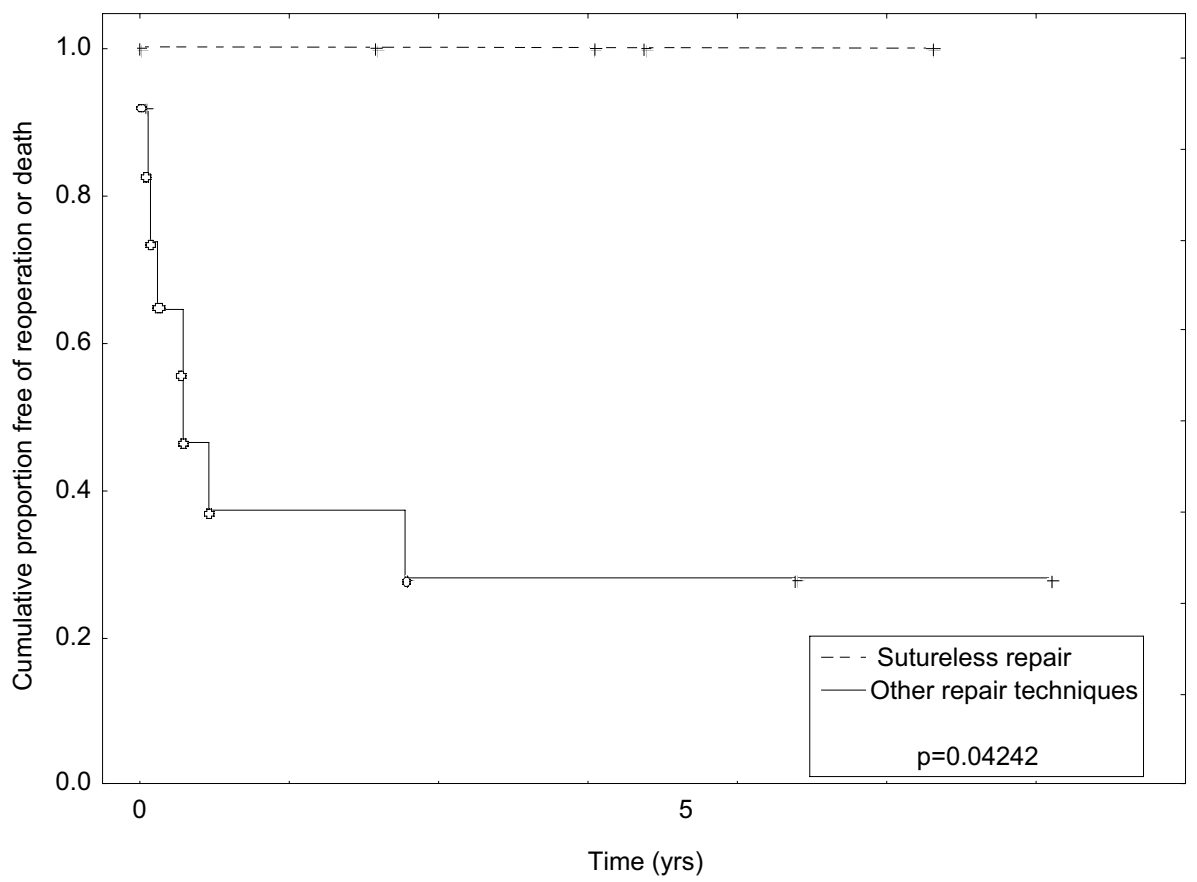

Figure 3. Unadjusted freedom from reoperation or death for patients with postrepair PVS stratified by sutureless technique versus other surgical techniques. Vertical marks, Censored events; circles, uncensored events.

vious reports, ${ }^{2,8}$ the indications for this technique in our institution have evolved beyond the original indication of postrepair PVS and now extend to patients without previous cardiac operations and patients at risk for PVS because of the presence of small pulmonary veins. ${ }^{11}$ In the present study, when controlling for other important variables, support for the sutureless technique is encouraging but not definitive. Nevertheless, the technique has a favorable safety profile in the postoperative and nonoperated mediastinum. In addition, technical problems, such as limited exposure and bleeding, can be controlled with simple maneu- vers (eg, division of the inferior vena cava and intrapleural hilar reapproximation).

The finding that the PVS score was the only statistically significant predictor of reoperation or death is interesting in that it might more accurately reflect the pulmonary venous pathology in comparison with our previous reports with a unilateral-bilateral stenosis classification scheme. ${ }^{2}$ The PVS score used in the present study ignores the presence of anastomotic stenosis, which might reflect surgical imperfection at the anastomosis rather than diffuse pulmonary venous pathology. Instead, the PVS score assesses the indi- 


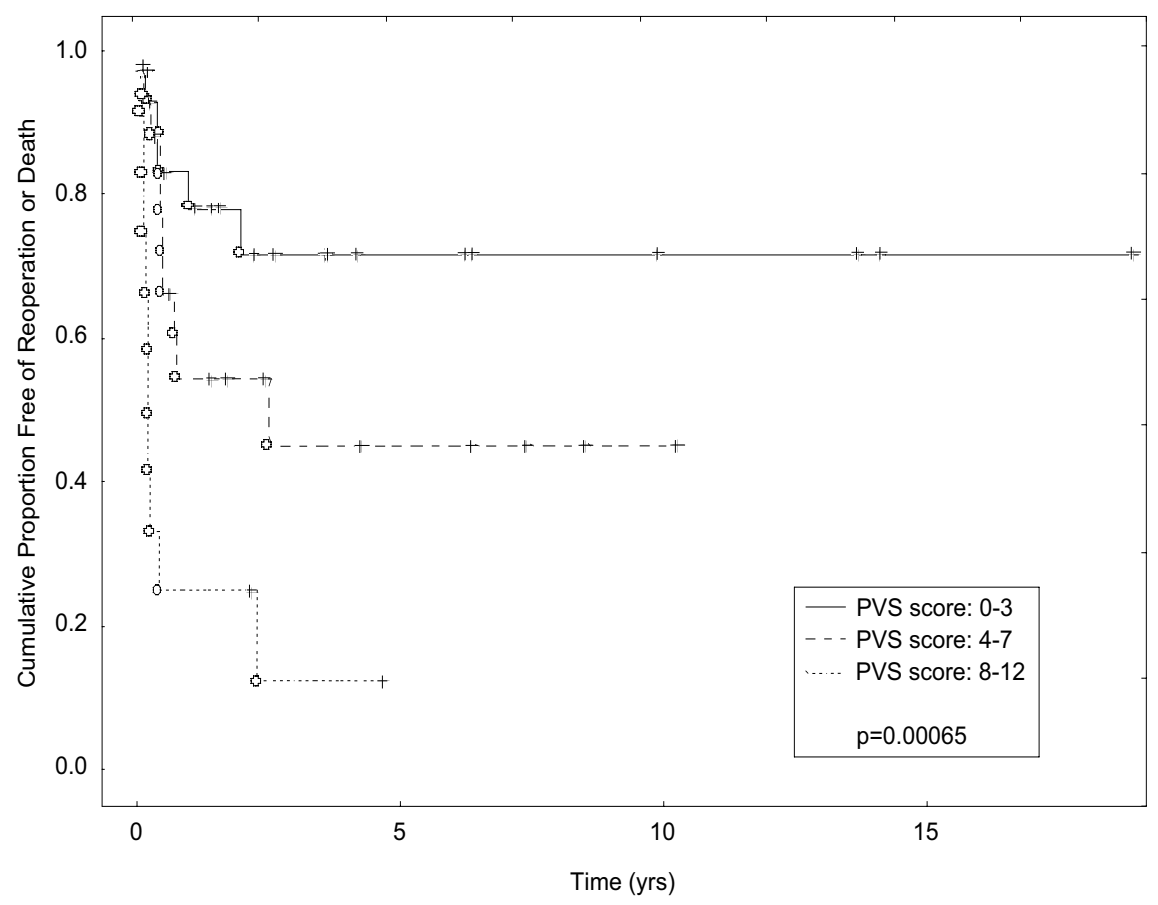

Figure 4. Freedom from reoperation or death stratified by PVS score. Vertical marks, censored events; circles, uncensored events.

vidual pulmonary veins and might better reflect pulmonary venous pathology. This rationale is very similar to the use of the pulmonary vein diameter sum as a predictor of recurrent PVS, as reported by Jenkins and associates. ${ }^{11}$

Because of the small sample size and the diversity of indication categories and operative techniques, the ability to draw conclusions is limited. On the basis of the presented data, the sample size needed to test the hypothesis that the sutureless technique reduces the risk of reoperation or death by $50 \%$ across the range of indication categories analyzed in the present study is approximately 150 patients in the setting of a randomized trial. In the setting of a retrospective study, however, where adjustment for baseline differences is required, a larger sample size would be required. Consequently, our ability to prove that the sutureless technique is advantageous might require more than doubling the size of our patient cohort, which will require 10 years at present rates of patient accrual.

An alternative strategy would be to limit analysis to the group of patients with the greatest risk of recurrent PVS and thereby increase the likelihood of detecting a benefit with the sutureless technique (if present). In subgroup analysis the postrepair PVS group seemed to derive the most benefit from sutureless repair, a finding that is consistent with the findings of others and might reflect the high rate of restenosis (and benefit) in this group of patients. ${ }^{4-6}$ Consequently, registry data from multiple sites selecting a high- risk population (eg, postrepair PVS) and allowing analysis of a large patient population is likely to be an expedient approach to validation of the sutureless technique. Data derived from the present study suggest that registry data should be adjusted for the extent of PVS (eg, PVS score).

The rationale for extending the use of the sutureless technique for the patients with small pulmonary veins believed to be at risk for future stenosis is based on a reasonable safety profile and reports that small pulmonary veins are important risk factors for later development of PVS after conventional total anomalous pulmonary venous drainage repair. ${ }^{11,13,14}$ In this regard we have recently changed our institutional strategy for the management of right atrial isomerism-total anomalous pulmonary venous drainage in patients undergoing single-ventricle palliation to primary sutureless repair. To demonstrate that a prophylactic primary sutureless repair for this subset of patients is efficacious will also require a very large patient cohort and is beyond the scope of the present study. Again, registry data might be the most expedient approach to evaluation of this strategy.

An important limitation of the study is that the PVS score is subjective in the grading of PVS and relies on retrospective inspection of echocardiographic and operative notes. In our current practice we rely heavily on high-resolution echocardiographic and magnetic resonance imaging data to allow more objective prospective grading of pulmonary 
vein pathology. Other weaknesses of the PVS score include the unavoidable loss of data with an ordinal variable (in contrast to a continuous variable), the lack of differentiation between localized and diffuse hypoplasia, and the lack of consideration of bilateral disease (eg, unilateral severe disease with a score of 4 is considered identical to bilateral mild disease with a score of 4 ).

Another important limitation of the present study is the small sample size, which leads to the use of a combined end point (reoperation or death) in the statistical analysis. Although this method improves the statistical power of the study, the outcomes cannot be considered equivalent.

As our patient population grows, we anticipate development of more robust statistical models to identify important predictors of success and failure in the management of this complex group of patients. With identification of these predictors, prophylactic strategies can be more expediently tested in newly identified high-risk populations, and management strategies can be modified for individual patients.

\section{References}

1. Caldarone CA, Najm HK, Kadletz M, Smallhorn JF, Freedom RM, Williams WG, et al. Surgical Management of total anomalous pulmonary venous drainage: impact of coexisting cardiac anomalies. Ann Thorac Surg. 1998;66:1521-6.

2. Caldarone CA, Najm HK, Kadletz M, Smallhorn JF, Freedom RM, Williams WG, et al. Relentless pulmonary vein stenosis after repair of total anomalous pulmonary venous drainage. Ann Thorac Surg. 1998; 66:1514-20

3. Coles JG, Yemets I, Najm HK, Lukanich JM, Perron J, Wilson GJ, et al. Experience with repair of congenital heart defects using adjunctive endovascular devices. J Thorac Cardiovasc Surg. 1995; 110:1513-20.

4. Ricci M, Elliot M, Cohen GA, Catalan G, Stark J, de Leval MR, et al. Management of pulmonary venous obstruction after correction of TAPVC: risk factors for adverse outcome. Eur J Cardiothorac Surg. 1999; 15:735-41.

5. Spray TL, Bridges ND. Surgical management of congenital and acquired pulmonary vein stenosis. Semin Thorac Cardiovasc Surg Pediatr Card Surg Annu. 1999;2:177-88.

6. Lacour-Gayet F, Zofhbi J, Serraf AE, Belli E, Piot D, Rey C, et al. Surgical management of progressive pulmonary venous obstruction after repair of total anomalous pulmonary venous connection. $J$ Thorac Cardiovasc Surg. 1999;117:679-87.

7. Lacour-Gayet F, Rey C, Planche C. Pulmonary vein stenosis. Description of a sutureless surgical procedure using the pericardium in situ. Arch Mal Coeur Vaiss. 1996;89:633-6.

8. Najm HK, Caldarone CA, Smallhorn J, Coles JG. A sutureless technique for the relief of pulmonary vein stenosis with the use of in situ pericardium. J Thorac Cardiovasc Surg. 1998;115:468-70.

9. LaBourene JI, Coles JG, Johnson DJ, Mehra A, Keeley FW, Rabinovitch $M$. Alterations in elastin and collagen related to the mechanism of progressive pulmonary venous obstruction in a piglet model. A hemodynamic, ultrastructural, and biochemical study. Circ Res. 1990; 66:438-56.

10. Sadr IM, Tan PE, Kieran MW, Jenkins KJ. Mechanism of pulmonary vein stenosis in infants with normally connected veins. Am J Cardiol. 2000;86:577-9.

11. Jenkins KJ, Sanders SP, Orav EJ, Coleman EA, Mayer JE, Colan SD. Individual pulmonary vein size and survival in infants with totally anomalous pulmonary venous connection. J Am Coll Cardiol. 1993; 22:201-6.

12. Pacifico AD, Mandke NV, McGrath LB, Colvin EV, Bini RM, Bargeron LM. Repair of congenital pulmonary venous stenosis with living autologous atrial tissue. $J$ Thorac Cardiovasc Surg. 1985;89:604-9.

13. Freedom RM, Hashmi A. Total anomalous pulmonary venous connections and consideration of the Fontan or one-ventricle repair. Ann Thorac Surg. 1998;66:681-2.

14. Hashmi A, Abu-Sulaiman R, McCrindle BW, Smallhorn JF, Williams WG, Freedom RM. Management and outcome of right isomerism: a 26 year experience. J Am Coll Cardiol. 1998;31:1120-6.

\section{Discussion}

Dr Francois Lacour-Gayet (Denver, Colo). Dr Tai-Jin Yun, I congratulate you for this excellent presentation that reports one of the largest series of PVS repair ever published.

As you said in your article, pulmonary vein surgery is dangerous. I will add that it can be frustrating.

Your study had very interesting information. You identify a new type of PVS occurring after nonpulmonary vein surgery. We have also seen this concerning group of patients.

You confirmed that the sutureless repair can be extended safely to native PVS conducted into the adhesion-free pericardial cavity. Your pulmonary venous score in 4 grades described very well the large spectrum of the disease. In this regard we have recently seen very severe forms with total pulmonary venous obstruction of one lung in which the patency of the pulmonary venous trunk could only be documented on magnetic resonance imaging.

Your article seems to recognize also some benefits to other techniques. My personal experience has been very much in favor of the sutureless repair. Since April 1995, when I first used this technique, I have not used another method. I thus far have not had any hemorrhagic complications and treat the left pulmonary vein from inside the left atrium. I resect totally the pulmonary venous lesion.

I would like to ask you the 3 following questions.

First, when dealing with a stenosis, I understand that you prefer to leave in place the lesion and open it longitudinally more than totally resect it. Could you comment on this?

Second, you have identified a new type of acquired PVS after nonpulmonary vein cardiac surgery. Could you comment on the cause of this pathology, which I have also observed? Could it be that the intraoperative traumatic process is a cause?

Finally, when there is a total pulmonary venous obstruction with a blind lung that corresponds, I understand, to your grade 3, do you consider that it is too late to do anything, or will you still go on and try to fix the stenosis to recover the lung?

Dr Yun. Thank you, Dr Lacour-Gayet. Regarding your first question of whether we excise or incise the stenotic vein, the decision is made on the basis of the operative findings rather than institutional strategy. We try to excise as much tissue as possible when it looks unhealthy and might have some proliferative potential. If the stenosis is too close to the hilar area, however, excessive excision is dangerous and might lead to intractable intrapleural bleeding.

Regarding your second question, we have 10 patients who had PVS after various cardiac operations. We classified those patients as the postoperative group rather than the postrepair group because these patients did not have congenital abnormalities of the pulmonary veins. These patients had cardiac operations that were not specifically dedicated to the pulmonary veins, but this does not necessarily mean that the primary operation has nothing to do with the development of PVS. For example, one patient had ventricular 
septal defect repair with a left atrial vent in the right upper pulmonary vein and had right upper PVS. We did a sutureless repair for right-sided pulmonary veins. During follow-up, the patient had recurrent PVS in the left side, which necessitated a second sutureless repair. The first PVS was presumably acquired disease, but the second PVS is of unclear cause, although it suggests an intrinsic abnormality in the pulmonary vein response to contralateral PVS.

Regarding your third question, I would say the indication for operation for the individual pulmonary vein might be different from the indication for operation for the patient. If you have a patient who has one atretic vein and 2 stenotic veins, there should not be any hesitation to open up the atretic pulmonary vein. But if you have a patient who has one atretic vein with mild symptoms and mildly increased right ventricular pressure, that is going to be a tough decision regarding the indication for operation. In our series we have some patients who had atretic pulmonary veins that were opened up. The end point of our observation was, however, reoperation or death and not the obstruction of the pulmonary veins we worked on, and we did not analyze the fate of the pulmonary vein after the operation.

Dr Thomas L. Spray (Philadelphia, $\mathrm{Pa}$ ). One of the real issues here is the pulmonary artery pressure or right ventricular pressure before you do this procedure. If the mean pulmonary vein score is somewhere in the neighborhood of 6 or 7 , that means that potentially 2 of the veins are open and 2 are closed. That might or might not be associated with pulmonary hypertension. One of the real concerns in distinguishing between what has been considered congenital PVS or veno-occlusive disease and either unilateral PVS, which can be congenital, or postrepair vein stenosis is determining the pulmonary artery pressure. If you have 1 or 2 veins that are unaffected and yet your pulmonary artery pressure is systemic or higher, that portends a worse overall long-term outcome, no matter what you do with the pulmonary vein repair.

Therefore the question I have to ask you is as follows: What were the right ventricular pressures in these patients in general, and then how did they respond to the repair, whether the vein stayed open or not?

Dr Yun. We have all echocardiographic data for our patients, but we did not include right ventricular pressure or gradient in the stenotic portion of the pulmonary vein in our analysis because of the heterogeneity of the patients. Right ventricular pressure might be useful for the risk factor analysis or outcome interpretation in some patient groups, such as postrepair PVS or congenital PVS with biventricular physiology. But right ventricular pressure cannot be applied to single-ventricle physiology or some patients who have biventricular physiology with an intracardiac shunt. In patients who had sutureless repair for postrepair PVS, right ventricular pressure was normalized after the operation and has been kept normal in all patients. In the congenital PVS group we have 18 patients with 6 reoperation deaths. In the remaining 12 patients, about one third of patients have a right ventricular/left ventricular pressure ratio of greater than 0.5 .

Dr Spray. But presumably the ones who died might have had the higher pulmonary artery pressures that did not respond to your treatment. That is one implication of the reasonably high mortality.

Dr Yun. Yes.

Dr Carl L. Backer (Chicago, Ill). I have a quick technical question. In this operation the suture line comes very close to both the left and right phrenic nerves. Do you have data about whether there was phrenic nerve injury in any of these patients? Do you have any tips to help avoid the phrenic nerve, which, again, is very close to that suture line?

Dr Yun. We tried to keep our suture line far posterior to the phrenic nerve. If we do the sutureless repair as a primary operation without any previous operation, we can see the course of the phrenic nerve very easily from inside because the pericardium is semitransparent. Furthermore, we open up the pleura on both sides when we do bilateral sutureless repair for 2 reasons: first, to check the course of the phrenic nerve from outside as well, and second, to check whether there is any intrapleural bleeding after completion of the procedure. If we do the sutureless repair in patients who have previous cardiac operations, then we have to check the course of the phrenic nerve from outside before we place a suture on the pericardium.

Dr Felix Haas (Muenchen, Germany). This is, indeed, a very interesting and useful technique to relieve PVS.

In my personal experience I believe that the right side is much easier to do than the left side. Did you find any differences between the left and right sides in terms of outcome or in terms of restenosis?

Dr Yun. Well, yes, we had some difficulties when we did the left-side sutureless repair. But recently we began to divide the inferior vena cava and lift up the heart toward the right side. After this technical modification, we did not have any difficulties in doing the left side. Divisions of the inferior vena cava is a very useful technique for bilateral sutureless repair as well. 